\title{
ISO spectroscopy of nearby starburst galaxies
}

\author{
Dietmar Kunze, Dieter Lutz, Dimitra Rigopoulou, \\ Michelle D. Thornley, and Reinhard Genzel \\ Max-Planck-Institut für extraterrestrische Physik, \\ D-85740 Garching, Germany
}

\begin{abstract}
We present spectroscopic mid-IR observations of prominent starburst galaxies obtained with the short wavelength spectrometer onboard the Infrared Space Observatory. The wavelength range accessible by ISO-sws (2.5$45 \mu \mathrm{m}$ ) contains a large number of emission lines of atomic and molecular hydrogen and several other atomic species. In this paper we discuss the interpretation of the [NeIII] $15.5 \mu \mathrm{m} /[\mathrm{NeII}] 12.8 \mu \mathrm{m}$ line-ratio, the faint [OIV] $25.9 \mu \mathrm{m}$ line and the pure rotational lines of molecular hydrogen observed in our target galaxies.
\end{abstract}

\section{The $[\mathrm{NeIII}] /[\mathrm{NeII}]$ line-ratio}

We have used ISO-sws to observe 16 starburst galaxies in the mid-IR. Beside the two starburst templates, M 82 and NGC 253, our sample contains nuclear starbursts in nearby $\mathrm{H}$ II galaxies and barred spirals, starburst rings and starbursts in merger systems. Due to the low extinction in the mid-IR the deeply embedded star-forming regions can be observed directly with ISO-swS.

The observed fine-structure line-ratios probe the density of the ionized gas and the hardness of the ionizing radiation field in our starburst galaxies (Kunze, 1998). The [NeIII] $15.5 \mu \mathrm{m} /[\mathrm{NeII}] 12.8 \mu \mathrm{m}$ ratio is of special interest, as it is fairly independent of chemical abundances, instrumental effects (same aperture and detectors) and extinction. The observed ratios of 0.05 to 10 together with population synthesis/photo-ionization calculations show a clear need for stars more massive than $30 \mathrm{M}_{\odot}$ in the starburst IMF. Higher upper mass cutoffs up to $100 \mathrm{M}_{\odot}$ cannot be ruled out, especially in case of short burst ages.

\section{Faint [OIV] emission}

A faint emission in the [OIV] $25.9 \mu \mathrm{m}$ high-excitation line has been detected in about $60 \%$ of our starburst galaxies (Lutz et al. 1998). The observation of this line is surprising, since it is not produced in measurable quantities in $\mathrm{H}$ II regions around hot main-sequence stars, which are the main energy source of starburst galaxies. SWS raster-observations along the major axis of M 82 show, that the [OIV] emission is spatially resolved and that the [OIV]-line radial velocities follow the CO 'rotation curve' by Götz et al. (1990). Various energy sources can be responsible for the faint [OIV] emission. In case of the extended [Orv] emission of $\mathrm{M} 82$, a weak AGN can be ruled out as energy source, because an AGN related origin would require a much larger [NeIII]/[NeII] ratio than observed. Similar arguments vote againts 'super-hot stars' with an extremely hard ionising 


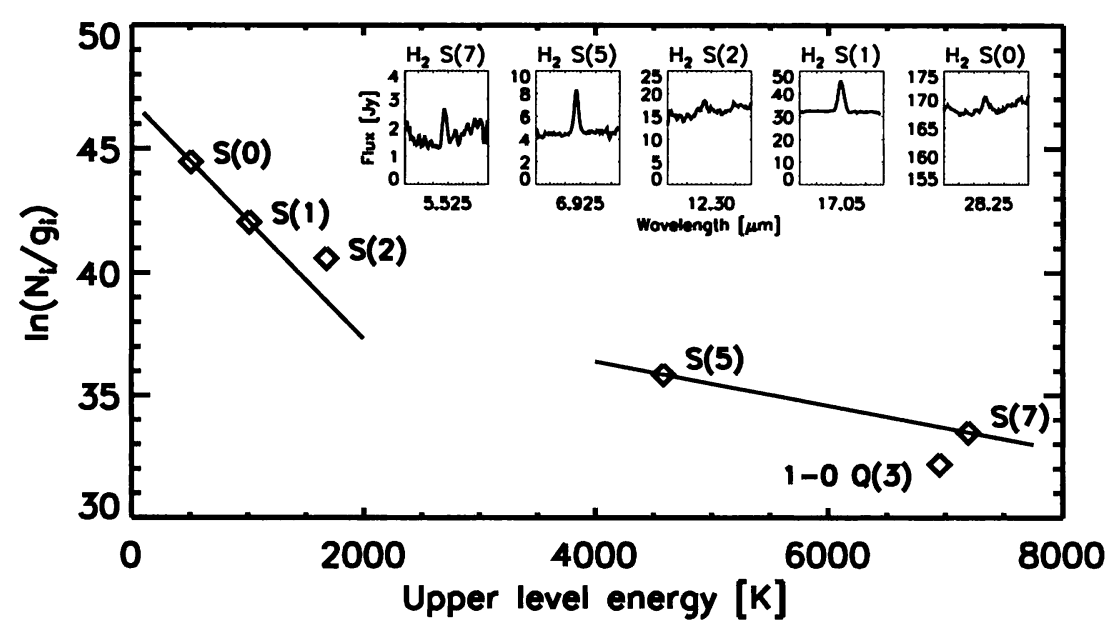

Figure 1. Excitation diagram of molecular hydrogen in NGC 253.

spectrum. The most plausible explanation for the faint [OIV] line is provided by ionizing shocks. Shock models with appropriate parameters and comparison with shock excited galactic templates (e.g., RCW 103) predict line intensities similar to the observed ones.

\section{Molecular hydrogen}

The pure rotational transitions of molecular hydrogen in starburst galaxies have been observed for the first time with ISO-sws. Excitation diagrams are used to derive temperatures for the emitting gas (see Fig. 1). The $\mathrm{H}_{2}(0-0) \mathrm{S}(1)$ line originates in our targets in warm molecular hydrogen of typically $120-200 \mathrm{~K}$. The $\mathrm{H}_{2}(0-0) \mathrm{S}(5)$ and $\mathrm{S}(7)$ lines trace a gas component with a higher excitation equivalent to a temperature of about $1000 \mathrm{~K}$ (up to $1700 \mathrm{~K}$ in IC 342). The warm component of the $\mathrm{H}_{2}$ gas accounts for $3-5 \%$ (up to $9 \%$ in M 82) of the total $\mathrm{CO}$ mass. The hotter component contributes much less. The $\mathrm{H}_{2}$ gas in the lower states might be partially excited by collisions due to the local kinetic temperature. The excitation of the higher states however is, as comparison with PDR models and observations of galactic templates (e.g., S 140, Timmermann et al. 1996) imply, dominated by UV fluorescence.

\section{References}

Götz, M., McKeith, C.D., Downes, D., Greve, A. 1990, A\&A 240, 52

Kunze, D., Genzel, R., Lutz, D., Spoon, H.W.W. 1998, in: J.L. Yun \& R. Liseau (eds.) Star Formation with the Infrared Space Observatory, ASP-CS 132, 378

Lutz, D., Kunze, D., Spoon, H.W.W., Thornley, M.D. 1998, A\&A 333, L75

Timmermann, R., Bertoldi, F., Wright, C.M., Drapatz, S., Draine, B.T., Haser, L., Sternberg, A. 1996, A\&A 315, L281 